\title{
Design ICCreatech semiconductor wafer accounting and probe measurement automatization software
}

\author{
Dmitry Argunov ${ }^{1,2}$, Boris Shiryaev ${ }^{1,2}$, Alexey Bezruk ${ }^{1}$, and Alexey Yushenko ${ }^{1}$ \\ ${ }^{1}$ Scientific-Research Institute of Semiconductor Devices JSC, 634034 Tomsk, Russia \\ ${ }^{2}$ Tomsk State University of Control Systems and Radioelectronics, 634050 Tomsk, Russia
}

\begin{abstract}
The different manufacturing processes automatization and creating a modern digital document transferring system is an important problem for enhancing factory performance. This paper presents the results of developing an automated information system for manufacturing monolithic integrated circuits. Server and client solutions have been developed supported features of electronic covering documents, making automated on-wafer electrical characteristics measurement, and measurement data analysis.
\end{abstract}

\section{Introduction}

Different information processing tasks automatization takes a leading part in the manufacturing industry. Work effectiveness questions, production quality, and safety improvement and reduction of human factors appear in conditions constantly upgrowth and complicating the manufactured devices and products.

For the Scientific-Research Institute of Semiconductor Devices, one of the main activity spheres is the sphere of microwave monolithic integrated circuits (MMIC) developing and manufacturing. For the on-wafer probe measurement of MMICs, there are several software solutions (Nucleus, Velox, MPI SENTIO, etc. [1-2]). However, they are not so much adapted for measurements related to developing new integrated circuits and to a greater extent purposed for well-established manufacturing. Also, these solutions don't support integration with a custom database or electronic document share system.

The main targets of developing are creating a software system that will provide tools for easy wafers tracking, measurement data collection and making different experiments. Designed solution should have several distinctive features:

1. Database support

2. System scaling feature

3. Flexible settings

4. Third-party measuring equipment integration features

5. User friendliness

Corresponding author: argunov_dp@niipp.ru 
A specialized software system has been developed that solves a stack of problems for automated on-wafer probe measurements and accounting covering documents in the MMIC fabrication process.

\section{System overview}

The developed software is a virtual distributed control system. It has a supervisory layer that built on three-layer multiunit client-server architecture. Server-side of the virtual control environment built on the two-layer system principle with its first relational database layer. The second outside layer is a business logic processor. The business logic layer has a modular structure with dedicated TCP/IP endpoints for each service module (fig. 1).

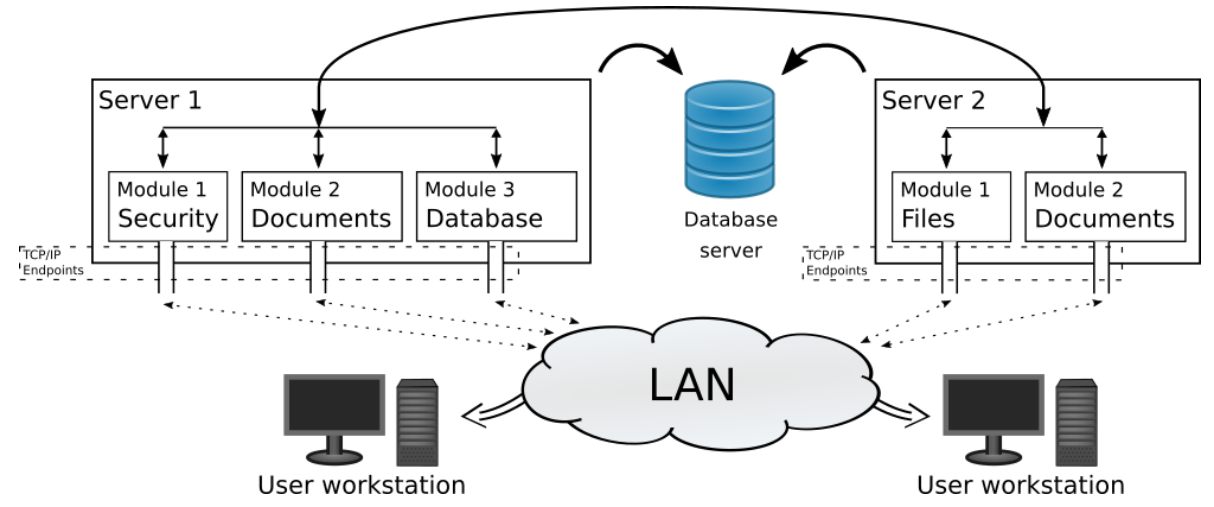

Fig. 1. Server-side architecture.

Each service module provides a set of methods and functions that can be called from the client-side. In addition, most modules can process connections from user applications independently from others. However, each module implements a special program interface for intercommunication. For example, the covering document processing module can request user authorization from the security module for executing limited document actions.

The modular structure of the outside system server layer is designed to easily upscale services calculation power capabilities. The server modules can be placed on different physical server machines for load balance.

A possible client connection structure is shown in figure 2 .

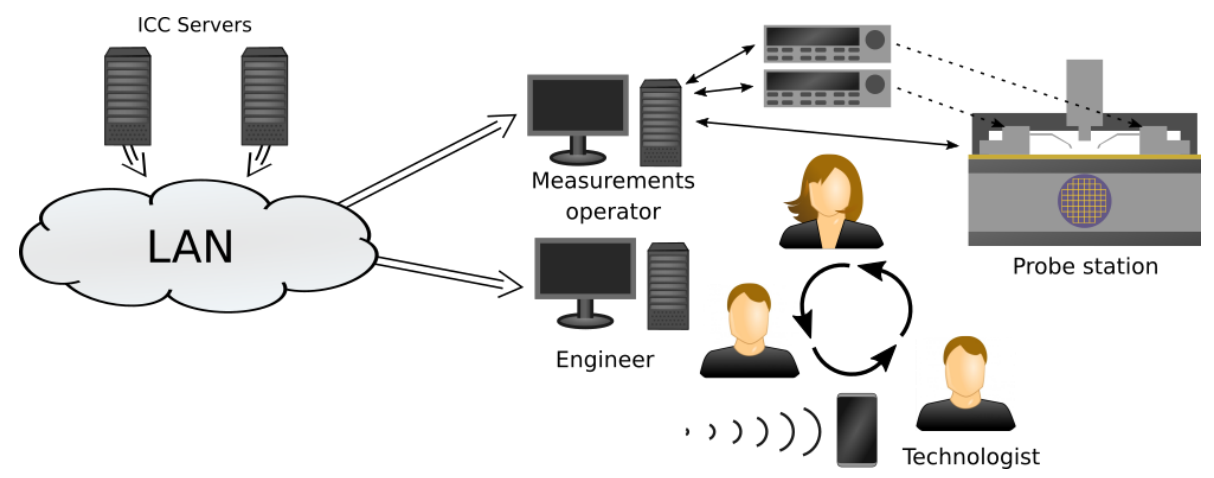

Fig. 2. Client communication scheme.

The client applications of the system are standalone-executable applications for Windows platform which can be started separately. However, for getting full features of the 
system the client applications must be started by the master client named "Software Distribution Client" (SDSC).

$S D S C$ - It is a service application for Windows desktops that worked on users' PCs in background mode. The application main purposes are authentication of a user personality in the system and distribute the newest versions of client applications on a workstation. The user interface of SDSC is shown in figure 3.
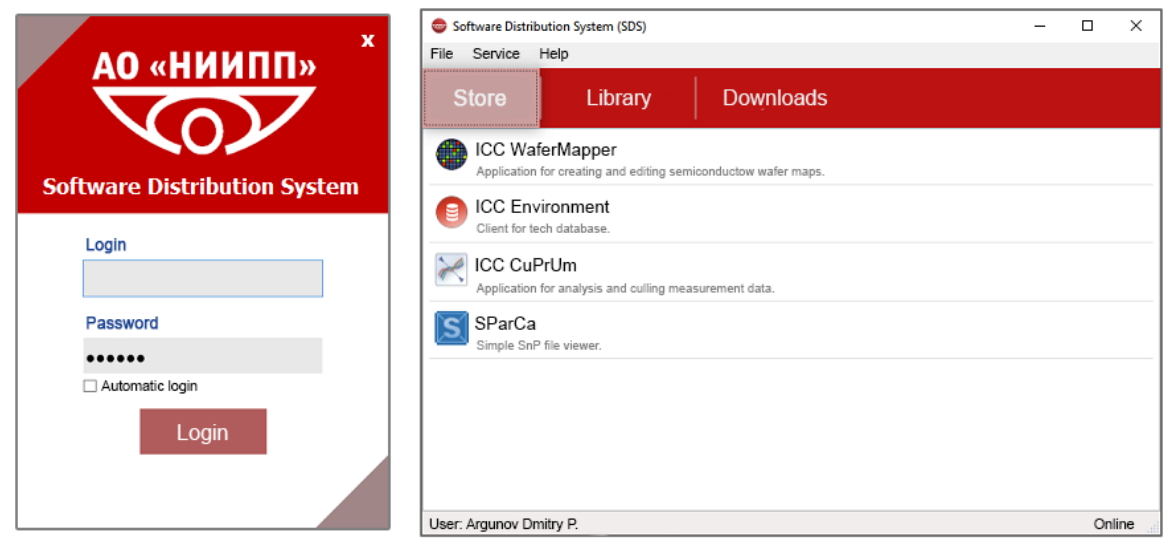

Fig. 3. SDSC user interface.

\section{User applications}

ICC Environment - application is purposed for interacting with the system information environment. It purposes manage digital converging documents for wafer on a route. Applications also have a table-based user interface for interacting with the database. It allows to keep an accounting of semiconductor materials (batches, epitaxial structures, individual semiconductor wafers), photomasks sets, technologies (technological operations, operation blocks, routes) and accompanying sheets. The application has a set of tools that make it easy to form a technological route, both from individual operations and ready-made operation blocks previously stored in the database. There is also functionality for flexible configuration of adaptive logical connections between operations and technology capabilities, followed by saving the route as a basic technology. Basic technologies make it easy to create new technological routes for the developed MMIC by simply selecting the capabilities required for the MMIC manufacture.

ICC WaferMapper - this program provides functionality for creating and editing semiconductor wafers maps for automated probe on-wafer measurements. It allows to create both simple uniformly filled maps with the same MMICs and maps with non-regular internal organization from topologically different MMICs. Created cards are stored in the database for future use.

ICC ProMeasurement - this application program is designed to automate the process of on-wafer probe measurement electrical parameters of individual MMICs (fig. 4). Semiautomated probe station must be used for on-wafer probe measurements executing. The application is responsible for sending commands to low-level probe station software and digital measurement equipment connected via Ethernet/USB/RS232. The program has a modular architecture based on dynamically connected .NET class libraries, build on managed extensibility framework (MEF). Modular architecture makes possible to expand the functionality, add support for additional peripheral and measuring equipment, as well as add new measurement automation plug-ins without changing the code and recompile of the program. 


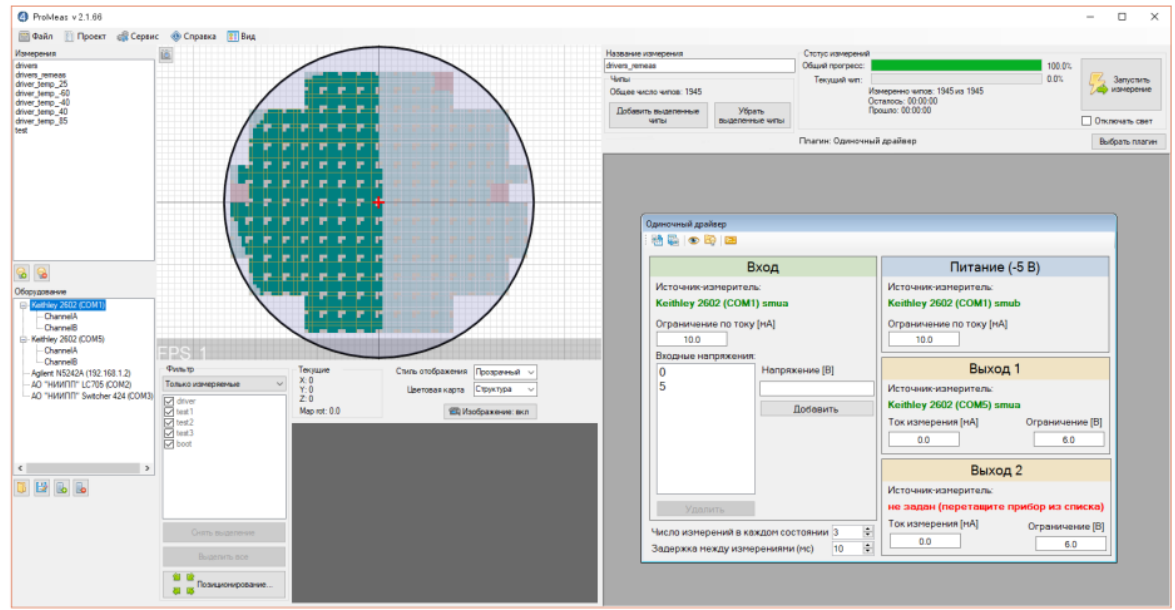

Fig. 4. Software for automated on-wafer probe measurements.

ICC CuPrUm - this program is intended for analysis, post-processing of electrical characteristics automated measurements results, the MMICs culling according the measured characteristics, followed by saving the valid map (fig. 5.). The dataset visualization system based on OpenGL [3] makes possible to display a large volume of measurement results without compromising program performance.

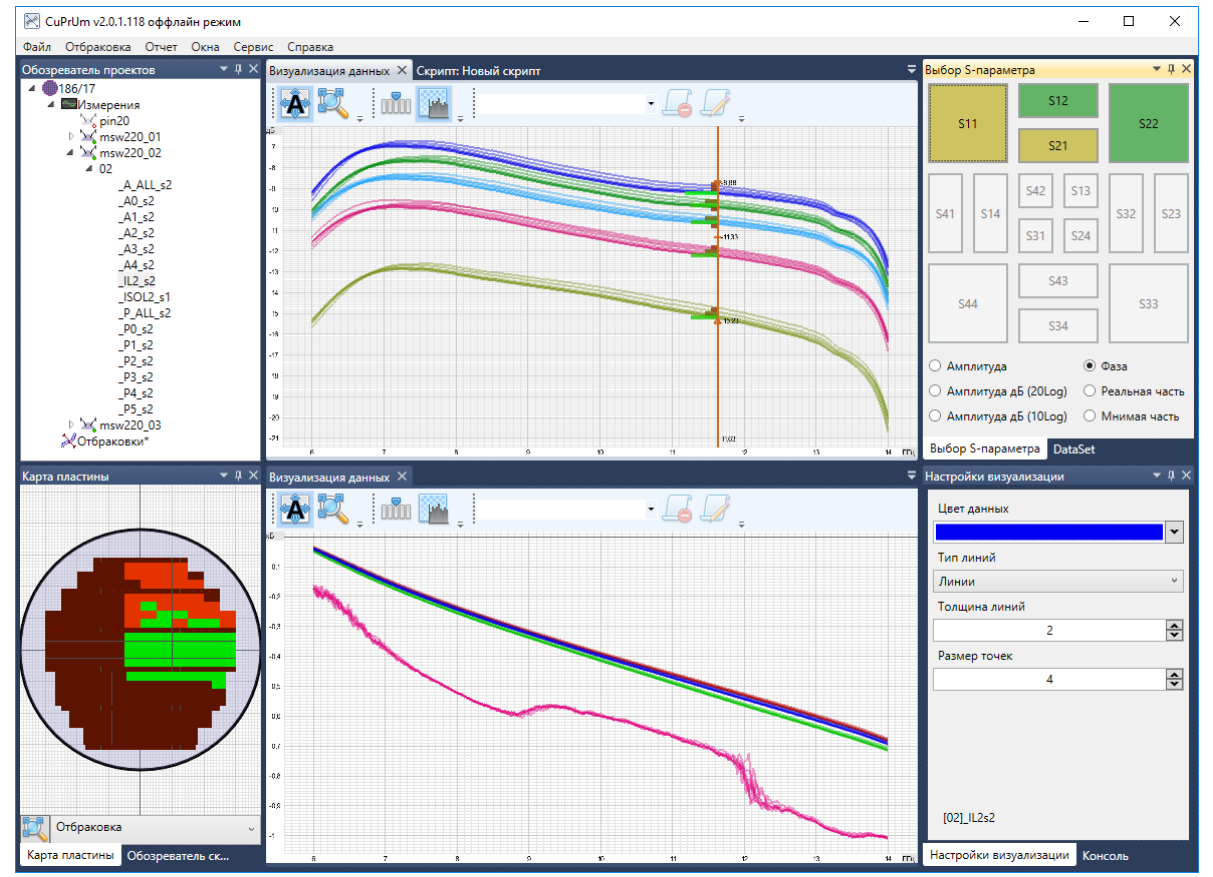

Fig. 5. CuPrUm user interface

For data post-processing has been developed a unique visual script system. In these scripts raw data presented by input node, and displayed data is shown by output node [4]. All mathematical data transforms presented by a visual block that has an input and output ports specific type. Output and input ports of these blocks can be connected if they have identic types. Simple example of script is shown in figure 6 . 


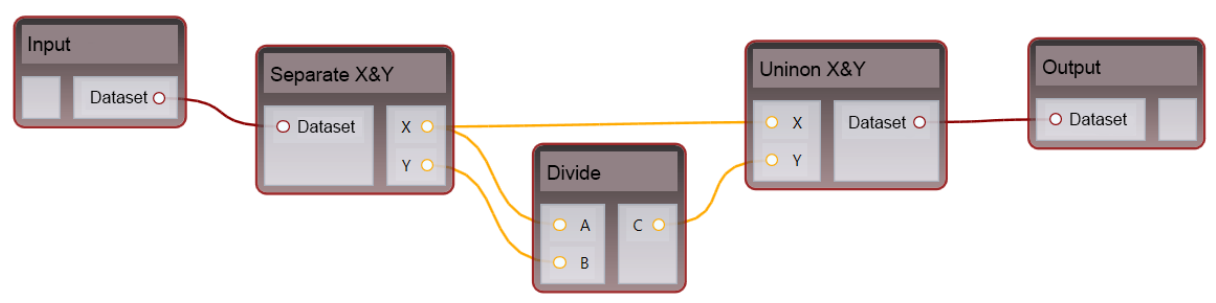

Fig. 6. CuPrUm visual script example.

This approach to data transformation by combination linked visual blocks is very efficient in practice [5]. Persons who do not have experience in the classical approach to programming easily study this script system.

Often when analyzing the characteristics there is a need to assess the measured data disperse, this assessment is not always possible to carry out visually from the totality of graphs. To assess the characteristics dispersion, a histogram tool is implemented in the software module (fig. 7).

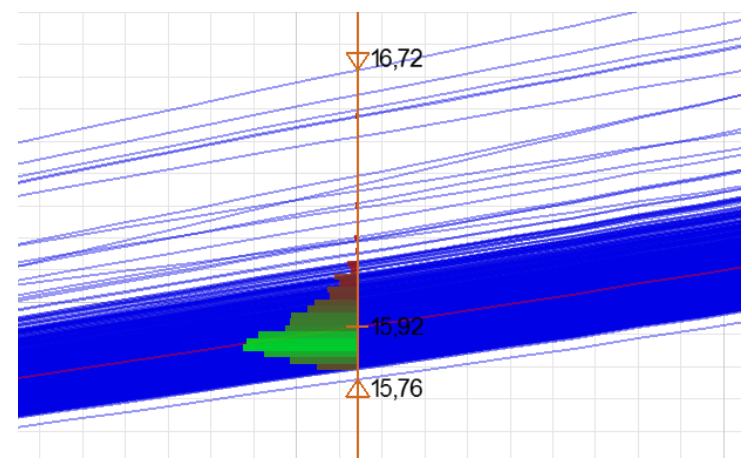

Fig. 7. Histogram tool in CuPrUm dataset viewer

This tool works in conjunction with markers; when it is activated, a histogram is added to the marker, showing the relative density of characteristics intersection points distribution with the marker line, divided into intervals.

ICC Technologist - the Android platform application. The main purpose is to provide access to system information resources for mobile employees, such as technologists and operators of various technological and measuring posts.

\section{Observations and conclusions}

In conclusion, it should be noted that the result of developed software implementation at the Scientific-Research Institute of Semiconductor Devices, the measurement processes were successfully automated, and the processing of the electrical characteristics measurement results of the manufactured MMIC output and intermediate control was optimized. At the moment work is underway to integrate the electronic circulation of covering documents and expand the user application modules functionality.

\section{References}

1. https://www.cascademicrotech.com/files/NUCLEUS_DS.pdf (20.08.2019)

2. http://www.mpi-corporation.com/ast/engineering-probe-systems/mpi-sentio-softwaresuite (21.08.2019). 
3. A. I. Evchinko, Open $G L$ and DirectX: graphics programming. For professionals (2006)

4. M. Wesley, Advances in Dataflow Programming Languages, ACM Computing Surveys, v. 1, pp. 1-34 (2004)

5. 4GL-Technologies. Basics of visual http://bourabai.kz/einf/4gl.htm (12.08.2019) 